\title{
MULTIPLE NONSEMITRIVIAL SOLUTIONS FOR A CLASS OF DEGENERATE QUASILINEAR ELLIPTIC SYSTEMS
}

\author{
Ghasem A. Afrouzi - Armin Hadjian
}

NicolaOs B. Zographopoulos

\begin{abstract}
We prove the existence of multiple nonnegative nonsemitrivial solutions for a degenerate quasilinear elliptic system. Our technical approach is based on variational methods.
\end{abstract}

\section{Introduction}

In this paper, we prove the multiplicity of solutions for the following degenerate quasilinear elliptic system, defined on $\Omega$,

$$
\begin{aligned}
-\nabla\left(\nu_{1}(x)|\nabla u|^{p-2} \nabla u\right)= & \lambda a(x)|u|^{p-2} u+\lambda b(x)|u|^{\alpha}|v|^{\beta} v \\
& +\frac{\mu(x)}{(\alpha+1)(\delta+1)}|u|^{\gamma-1}|v|^{\delta+1} u, \\
-\nabla\left(\nu_{2}(x)|\nabla v|^{q-2} \nabla v\right)= & \lambda d(x)|v|^{q-2} v+\lambda b(x)|u|^{\alpha}|v|^{\beta} u \\
& +\frac{\mu(x)}{(\beta+1)(\gamma+1)}|u|^{\gamma+1}|v|^{\delta-1} v,
\end{aligned}
$$

$$
\left.u\right|_{\partial \Omega}=\left.v\right|_{\partial \Omega}=0,
$$

2010 Mathematics Subject Classification. 35J70, 35P30, 35B32, 35J50.

Key words and phrases. Degenerate equations, principal eigenvalue, multiple solutions, bifurcation, Ekeland's variational principle. 
where $\Omega$ is a bounded and connected subset of $\mathbb{R}^{N}, N \geq 2$. The degeneracy of this system is considered in the sense that the measurable, nonnegative diffusion coefficients $\nu_{1}, \nu_{2}$ are allowed to vanish in $\Omega$, (as well as at the boundary $\partial \Omega$ ) and/or to blow up in $\bar{\Omega}$.

Throughout this paper, we assume the following hypotheses:

$(\mathcal{H}) N>p>1, N>q>1, \alpha \geq 0$ and $\beta \geq 0$ satisfying $(\alpha+1) / p+$ $(\beta+1) / q=1, \gamma \geq 0, \delta \geq 0$ and $p<\gamma+1$ or $q<\delta+1$ satisfying $(\gamma+1) / p^{*}+(\delta+1) / q^{*}<1$.

The quantities $p^{*}$ and $q^{*}$ are defined in the next section.

$\left(\mathcal{H}_{1}\right)$ The exponents $\alpha, \beta, \gamma$ and $\delta$ satisfy also the general condition

$$
\frac{1}{(\alpha+1)(\delta+1)}+\frac{1}{(\beta+1)(\gamma+1)}<1 \text {. }
$$

We introduce the function space

$(\mathcal{N})_{p}$ which consists of nonnegative weighted functions $\nu: \Omega \subset \mathbb{R}^{N} \rightarrow \mathbb{R}$ such that $\nu$ vanishes and/or tends to infinity at finite points at most, $\nu \in$ $L_{\mathrm{loc}}^{1}(\Omega), \nu^{-1 /(p-1)} \in L_{\mathrm{loc}}^{1}(\Omega)$ and $\nu^{-s} \in L^{1}(\Omega)$, for some $p>1, s>$ $\max \{N / p, 1 /(p-1)\}$ satisfying $p s \leq N(s+1)$.

Then for the weight functions $\nu_{1}, \nu_{2}$ we assume the following hypothesis:

$(\mathcal{N})$ There exist functions $\mu_{1}$ satisfying condition $(\mathcal{N})_{p}$, for some $s_{p}$, and $\mu_{2}$ satisfying condition $(\mathcal{N})_{q}$, for some $s_{q}$, such that

$$
\frac{\mu_{1}(x)}{c_{1}} \leq \nu_{1}(x) \leq c_{1} \mu_{1}(x) \text { and } \frac{\mu_{2}(x)}{c_{2}} \leq \nu_{2}(x) \leq c_{2} \mu_{2}(x),
$$

almost everywhere in $\Omega$, for some constants $c_{1}>1$ and $c_{2}>1$.

Furthermore, we suppose that the coefficient functions $a(x), d(x), b(x)$ and $\mu(x)$ satisfy the following conditions:

$\left(\Upsilon_{1}\right) a$ is a smooth function, at least $C_{\text {loc }}^{0, \zeta}(\Omega)$, for some $\zeta \in(0,1)$, such that $a \in L^{p^{*} /\left(p^{*}-p\right)}(\Omega)$ and either there exists $\Omega_{a}^{+} \subset \Omega$ of positive Lebesgue measure, i.e. $\left|\Omega_{a}^{+}\right|>0$ such that $a(x)>0$, for all $x \in \Omega_{a}^{+}$, neither $a(x) \equiv 0$, in $\Omega$.

$\left(\Upsilon_{2}\right) d$ is a smooth function, at least $C_{\text {loc }}^{0, \zeta}(\Omega)$, for some $\zeta \in(0,1)$, such that $d \in L^{q^{*}} /\left(q^{*}-q\right)(\Omega)$ and either there exists $\Omega_{d}^{+} \subset \Omega$ of positive Lebesgue measure, i.e. $\left|\Omega_{d}^{+}\right|>0$ such that $d(x)>0$, for all $x \in \Omega_{d}^{+}$, neither $d(x) \equiv 0$ in $\Omega$.

$\left(\Upsilon_{3}\right) b(x) \geq 0$ almost everywhere in $\Omega, b \not \equiv 0$ and $b \in L^{\omega_{1}}(\Omega) \cap L^{\infty}(\Omega)$, where $\omega_{1}=\left[1-(\alpha+1) / p^{*}-(\beta+1) / q^{*}\right]^{-1}$.

$\left(\Upsilon_{4}\right) \mu$ is sign changing (i.e. $\left.\mu^{+} \not \equiv 0, \mu^{-} \not \equiv 0\right)$ and $\mu \in L^{\omega_{2}}(\Omega) \cap L^{\infty}(\Omega)$, where $\omega_{2}=\left[1-(\gamma+1) / p^{*}-(\delta+1) / q^{*}\right]^{-1}$.

In addition the function $\mu(x)$ satisfies the following key condition: 
$\left(\Upsilon_{5}\right) \int_{\Omega} \mu(x)\left|u_{1}\right|^{\gamma+1}\left|v_{1}\right|^{\delta+1} d x<0$

where $\left(u_{1}, v_{1}\right)$ is the positive normalized eigenfunction of the unperturbed system:

$$
\begin{gathered}
-\nabla\left(\nu_{1}(x)|\nabla u|^{p-2} \nabla u\right)=\lambda a(x)|u|^{p-2} u+\lambda b(x)|u|^{\alpha}|v|^{\beta} v, \quad x \in \Omega, \\
-\nabla\left(\nu_{2}(x)|\nabla v|^{q-2} \nabla v\right)=\lambda d(x)|v|^{q-2} v+\lambda b(x)|u|^{\alpha}|v|^{\beta} u, \quad x \in \Omega, \\
\left.u\right|_{\partial \Omega}=\left.v\right|_{\partial \Omega}=0,
\end{gathered}
$$

corresponding to the positive principal eigenvalue $\lambda_{1}$.

As it was proved in [13] (see also Section 2, Theorem 2.4), system $\left(1.4_{\lambda}\right.$ ) is in fact an eigenvalue problem which admits a positive principal eigenvalue $\lambda_{1}$ and the corresponding normalized eigenfunctions $\left(u_{1}, v_{1}\right)$ are positive, up to singular and/or degenerate points, componentwise. Moreover, up to the singular/degenerate points of $\nu_{1}$ and $\nu_{2}$, they are also bounded and sufficiently smooth.

REMARK 1.1. An example of the weighted function $\mu(x)$ which satisfies both conditions $\left(\Upsilon_{4}\right)$ and $\left(\Upsilon_{5}\right)$ may be the following; Let $\mu(x)$ be a smooth function in $\bar{\Omega}$, which is zero at a neighbourhood of the singular/degenerate points and $\mu(x)$ satisfies

$$
\int_{\Omega} \mu^{-}(x)\left|u_{1}\right|^{\gamma+1}\left|v_{1}\right|^{\delta+1} d x>\int_{\Omega} \mu^{+}(x)\left|u_{1}\right|^{\gamma+1}\left|v_{1}\right|^{\delta+1} d x,
$$

for $\mu(x)=\mu^{+}(x)-\mu^{-}(x)$, i.e. $\mu^{+}$and $\mu^{-}$are the positive and the negative part of $\mu$, respectively. More precisely, let $z_{i}, i=1, \ldots, n$, be the finite singular and/or degenerate points. Assume for some small enough $\varepsilon$, the spheres $B_{\varepsilon}\left(z_{i}\right)$ centered at $z_{i}$. Since $z_{i}$ are finite we can find $\widetilde{\Omega} \subset \Omega$, such that

$$
\widetilde{\Omega} \cap \overline{\bigcup_{i} B_{\varepsilon}\left(z_{i}\right)}=\emptyset \text {. }
$$

Note that $\widetilde{\Omega}$ may be chosen such that, both $u_{i}$ and $v_{1}$ are uniformly bounded from above and uniformly bounded away from zero.

We define now $\mu$ to be continuous in $\bar{\Omega}$, such that $\mu(x)=0$, for $x \in \bigcup_{i} \overline{B_{\varepsilon}\left(z_{i}\right)}$, $\mu$ is positive in $\Omega \backslash \overline{\widetilde{\Omega} \cup \bigcup_{i} B_{\varepsilon}\left(z_{i}\right)}$, with $\mu(x)<\delta$, in $\Omega \backslash \overline{\widetilde{\Omega} \cup \bigcup_{i} B_{\varepsilon}\left(z_{i}\right)}$, $\delta$ small enough and $\mu$ is negative in $\widetilde{\Omega}$, with sufficiently large $L^{\infty}$ norm, such that

$$
\int_{\widetilde{\Omega}}|\mu(x)|\left|u_{1}\right|^{\gamma+1}\left|v_{1}\right|^{\delta+1} d x>\int_{\Omega \backslash \widetilde{\Omega}} \mu^{+}(x)\left|u_{1}\right|^{\gamma+1}\left|v_{1}\right|^{\delta+1} d x,
$$

i.e. (1.6) holds. 
An example of the physical motivation of the assumptions $(\mathcal{N}),(\mathcal{N})_{p}$ may be found in [4, p. 79]. These assumptions are related to the modelling of reaction diffusion processes in composite materials occupying a bounded domain $\Omega$, which at some points they behave as perfect insulators. When at some points the medium is perfectly insulating, it is natural to assume that $\nu_{1}(x)$ and/or $\nu_{2}(x)$ vanish in $\bar{\Omega}$. For more information we refer to [13] and the references therein.

Multiplicity results for semilinear and quasilinear elliptic systems have received a great deal of interest in recent years; see, for instance, the papers [1]-[3], [5], [6], [8]-[10], [12] and the references therein.

We note that the procedure here is based on the arguments developed in [6] and [8]. Following along the same lines as in [8], we will prove multiplicity of nonsemitrivial solutions for the system $\left(1.1_{\lambda}\right)-\left(1.2_{\lambda}\right)$.

\section{The eigenvalue problem $\left(1.4_{\lambda}\right)-\left(1.5_{\lambda}\right)$}

Let $\nu(x)$ be a nonnegative weight function in $\Omega$ which satisfies condition $(\mathcal{N})_{p}$. We consider the weighted Sobolev space $\mathcal{D}_{0}^{1, p}(\Omega, \nu)$ to be defined as the closure of $C_{0}^{\infty}(\Omega)$ with respect to the norm

$$
\|u\|_{\mathcal{D}_{0}^{1, p}(\Omega, \nu)}:=\left(\int_{\Omega} \nu(x)|\nabla u|^{p}\right)^{1 / p} .
$$

The space $\mathcal{D}_{0}^{1, p}(\Omega, \nu)$ is a reflexive Banach space. For a discussion about the space setting we refer to [7] and the references therein. Let

$$
p_{s}^{*}:=\frac{N p s}{N(s+1)-p s} .
$$

Lemma 2.1. Assume that $\Omega$ is a bounded domain in $\mathbb{R}^{N}$ and the weight $\nu$ satisfies $(\mathcal{N})_{p}$. Then the following embeddings hold:

(a) $\mathcal{D}_{0}^{1, p}(\Omega, \nu) \hookrightarrow L^{p_{s}^{*}}(\Omega)$ continuously for $1<p_{s}^{*}<N$,

(b) $\mathcal{D}_{0}^{1, p}(\Omega, \nu) \hookrightarrow L^{r}(\Omega)$ compactly for any $r \in\left[1, p_{s}^{*}\right)$.

The space setting for our problem is the product space

$$
Z:=\mathcal{D}_{0}^{1, p}\left(\Omega, \nu_{1}\right) \times \mathcal{D}_{0}^{1, q}\left(\Omega, \nu_{2}\right)
$$

equipped with the norm

$$
\|z\|_{Z}:=\|u\|_{\mathcal{D}_{0}^{1, p}\left(\Omega, \nu_{1}\right)}+\|v\|_{\mathcal{D}_{0}^{1, q}\left(\Omega, \nu_{2}\right)}, \quad z=(u, v) \in Z .
$$

Observe that inequalities (1.3) in condition $(\mathcal{N})$ implies that the functional spaces $\mathcal{D}_{0}^{1, p}\left(\Omega, \nu_{1}\right) \times \mathcal{D}_{0}^{1, q}\left(\Omega, \nu_{2}\right)$ and $\mathcal{D}_{0}^{1, p}\left(\Omega, \mu_{1}\right) \times \mathcal{D}_{0}^{1, q}\left(\Omega, \mu_{2}\right)$ are equivalent.

In the sequel we denote by $p^{*}$ and $q^{*}$ the quantities $p_{s_{p}}^{*}$ and $p_{s_{q}}^{*}$, respectively, where $s_{p}$ and $s_{q}$ are induced by condition $(\mathcal{N})$. Also, we use $\|\cdot\|_{1, p}$ and $\|\cdot\|_{1, q}$ for the norms $\|\cdot\|_{\mathcal{D}_{0}^{1, p}\left(\Omega, \nu_{1}\right)}$ and $\|\cdot\|_{\mathcal{D}_{0}^{1, q}\left(\Omega, \nu_{2}\right)}$, respectively. 
We introduce the functionals $J, D, B, M: Z \rightarrow \mathbb{R}$ as follows:

$$
\begin{aligned}
J(u, v) & :=\frac{\alpha+1}{p} \int_{\Omega} \nu_{1}(x)|\nabla u|^{p} d x+\frac{\beta+1}{q} \int_{\Omega} \nu_{2}(x)|\nabla v|^{q} d x, \\
D(u, v) & :=\frac{\alpha+1}{p} \int_{\Omega} a(x)|u|^{p} d x+\frac{\beta+1}{q} \int_{\Omega} d(x)|v|^{q} d x \\
B(u, v) & :=\int_{\Omega} b(x)|u|^{\alpha+1}|v|^{\beta+1} d x \\
M(u, v) & :=\int_{\Omega} \mu(x)|u|^{\gamma+1}|v|^{\delta+1} d x .
\end{aligned}
$$

It is a standard procedure (see [8], [12]) to prove the following properties of these functionals.

Lemma 2.2. The functionals $J, D, B$, and $M$ are well defined. Moreover, $J$ is continuous and $D, B$ and $M$ are compact.

Next, we introduce the functionals $A_{\lambda}, I_{\lambda}: Z \rightarrow \mathbb{R}$ in the following way:

$$
\begin{aligned}
& A_{\lambda}(u, v):=J(u, v)-\lambda D(u, v)-\lambda B(u, v), \\
& I_{\lambda}(u, v):=A_{\lambda}(u, v)-\frac{1}{(\gamma+1)(\delta+1)} M(u, v) .
\end{aligned}
$$

The functionals $A_{\lambda}$ and $I_{\lambda}$ are well defined, and they are weakly lower semicontinuous. Clearly, $I_{\lambda} \in C^{1}(Z, \mathbb{R})$.

DeFinition 2.3. We say that $(u, v)$ is a weak solution of the system $\left(1.1_{\lambda}\right)-$ $\left(1.2_{\lambda}\right)$ if $(u, v)$ is a critical point of the functional $I_{\lambda}$, i.e.

$$
\begin{aligned}
\int_{\Omega} \nu_{1}(x)|\nabla u|^{p-2} \nabla u \cdot \nabla \phi d x= & \lambda \int_{\Omega} a(x)|u|^{p-2} u \phi d x+\lambda \int_{\Omega} b(x)|u|^{\alpha-1}|v|^{\beta+1} u \phi d x \\
& +\frac{1}{(\alpha+1)(\delta+1)} \int_{\Omega} \mu(x)|u|^{\gamma-1}|v|^{\delta+1} u \phi d x \\
\int_{\Omega} \nu_{2}(x)|\nabla v|^{q-2} \nabla v \cdot \nabla \psi d x= & \lambda \int_{\Omega} d(x)|v|^{q-2} v \psi d x+\lambda \int_{\Omega} b(x)|u|^{\alpha+1}|v|^{\beta-1} v \psi d x, \\
& +\frac{1}{(\beta+1)(\gamma+1)} \int_{\Omega} \mu(x)|u|^{\gamma+1}|v|^{\delta-1} v \psi d x
\end{aligned}
$$

for any $(\phi, \psi) \in Z$.

By a semitrivial solution, we mean any weak solution $(u, v) \in Z$ of the form $(u, 0)$ or $(0, v)$. Otherwise, the solution is called nonsemitrivial.

Concerning the eigenvalue problem $\left(1.4_{\lambda}\right)-\left(1.5_{\lambda}\right)$ we have the following result, which was proved in [13].

TheOrem 2.4 ([13, Theorem 1.1$])$. The system $\left(1.4_{\lambda}\right)-\left(1.5_{\lambda}\right)$ admits a positive principal eigenvalue $\lambda_{1}$, given by

$$
\lambda_{1}=\inf _{D(u, v)+B(u, v)=1} J(u, v) .
$$


The associated normalized eigenfunction $\left(u_{1}, v_{1}\right)$ belongs to $Z$ and each component is nonnegative. In addition,

(a) the set of all eigenfunctions corresponding to the principal eigenvalue $\lambda_{1}$ forms a one-dimensional manifold $E_{1} \subset Z$, which is defined by

$$
E_{1}=\left\{\left(c_{1} u_{1}, c_{1}^{p / q} v_{1}\right): c_{1} \in \mathbb{R}\right\} .
$$

(b) $\lambda_{1}$ is the only eigenvalue of $\left(1.4_{\lambda}\right)-\left(1.5_{\lambda}\right)$ to which corresponds a componentwise nonnegative eigenfunction.

(c) $\lambda_{1}$ is isolated in the following sense: there exists $\eta>0$, such that the interval $\left(0, \lambda_{1}+\eta\right)$ does not contain any other eigenvalue than $\lambda_{1}$.

Based on the properties of $\lambda_{1}$, the authors in [11], proved certain bifurcation results:

Definition 2.5. Let $E=\mathbb{R} \times Z$ be equipped with the norm

$$
\|(\lambda, u, v)\|_{E}=\left(|\lambda|^{2}+\|(u, v)\|_{Z}^{2}\right)^{1 / 2}, \quad(\lambda, u, v) \in E .
$$

We say that the set

$$
C=\left\{(\lambda, u, v) \in E:(\lambda, u, v) \text { solves }\left(1.1_{\lambda}\right),(u, v) \neq(0,0)\right\}
$$

is a continuum of nontrivial solutions of $\left(1.1_{\lambda}\right)$, if it is a connected set in $E$ with respect to the topology induced by the norm (2.2). We say $\lambda_{0} \in \mathbb{R}$ is a bifurcation point of the system $\left(1.1_{\lambda}\right)$ (in the sense of Rabinowitz), if there is a continuum of nontrivial solutions $C$ of $\left(1.1_{\lambda}\right)$ such that $\left(\lambda_{0}, 0,0\right) \in \bar{C}$ and $C$ is either unbounded in $E$ or there is an eigenvalue $\widehat{\lambda} \neq \lambda_{0}$, such that $(\widehat{\lambda}, 0,0) \in \bar{C}$.

More precisely, from [11, Theorem 4.6 and Proposition 4.7] we have that

THEOREM 2.6. The principal eigenvalue $\lambda_{1}>0$ of the unperturbed problem $\left(1.4_{\lambda}\right)-\left(1.5_{\lambda}\right)$ is a bifurcation point (in the sense of Rabinowitz) of the perturbed system $\left(1.1_{\lambda}\right)$. Moreover, there exists an $\eta>0$ small enough, such that for each $(\lambda, u, v) \in C \cap B_{\eta}\left(\lambda_{1}, 0\right)$, we have $u(x) \geq 0$ and $v(x) \geq 0$, almost everywhere in $\Omega$.

Based now on the properties of the scalar eigenvalue problem, we may prove the following properties of the solutions of $\left(1.1_{\lambda}\right)-\left(1.2_{\lambda}\right)$.

Lemma 2.7. Let $\lambda$ be close enough to $\lambda_{1}$. Every nontrivial solution $(u, v)$ of $\left(1.1_{\lambda}\right)-\left(1.2_{\lambda}\right)$ is nonsemitrivial.

ProOF. First consider the following eigenvalue problems:

$$
\begin{aligned}
-\nabla\left(\nu_{1}(x)|\nabla u|^{p-2} \nabla u\right) & =\lambda a(x)|u|^{p-2} u, \quad x \in \Omega, \\
\left.u\right|_{\partial \Omega} & =0,
\end{aligned}
$$


and

$$
\begin{aligned}
-\nabla\left(\nu_{2}(x)|\nabla v|^{q-2} \nabla v\right) & =\lambda d(x)|v|^{q-2} v, \quad x \in \Omega, \\
\left.v\right|_{\partial \Omega} & =0 .
\end{aligned}
$$

It is known (see [7]) that the problem $\left(2.3_{\lambda}\right)-\left(2.4_{\lambda}\right)\left(\left(2.5_{\lambda}\right)-\left(2.6_{\lambda}\right)\right.$, resp.) has a positive principal eigenvalue $\lambda^{\prime}\left(\lambda^{\prime \prime}\right.$, resp. $)$, which is characterized variationally by

$$
\begin{gathered}
\lambda^{\prime}=\inf _{u \in \mathcal{D}_{0}^{1, p}\left(\Omega, \nu_{1}\right) \backslash\{0\}} \frac{\int_{\Omega} \nu_{1}(x)|\nabla u|^{p} d x}{\int_{\Omega} a(x)|u|^{p} d x} \\
\left(\lambda^{\prime \prime}=\inf _{v \in \mathcal{D}_{0}^{1, q}\left(\Omega, \nu_{2}\right) \backslash\{0\}} \frac{\int_{\Omega} \nu_{2}(x)|\nabla v|^{q} d x}{\int_{\Omega} d(x)|v|^{q} d x}, \text { resp. }\right) .
\end{gathered}
$$

This eigenvalues is simple and isolated and it is the only one having a positive eigenfunction $\phi^{\prime}\left(\phi^{\prime \prime}\right.$, resp.). Now, observe that the nonzero component of any semitrivial solution of the system $\left(1.1_{\lambda}\right)-\left(1.2_{\lambda}\right)$ corresponds to an eigenfunction of $\left(2.3_{\lambda}\right)-\left(2.4_{\lambda}\right)$ or $\left(2.5_{\lambda}\right)-\left(2.6_{\lambda}\right)$. So it suffices to prove that $\lambda_{1}<\min \left\{\lambda^{\prime}, \lambda^{\prime \prime}\right\}$. Suppose not. Then the system $\left(2.4_{\lambda^{\prime}}\right)-\left(2.5_{\lambda^{\prime}}\right)\left(\left(2.4_{\lambda^{\prime \prime}}\right)-\left(2.5_{\lambda^{\prime \prime}}\right)\right.$, resp. $)$ would have a solution $\left(\phi^{\prime}, 0\right)\left(\left(0, \phi^{\prime \prime}\right)\right.$, resp.). From the variational characterization (2.1) of the eigenvalue $\lambda_{1}$ this is a contradiction, and so the proof is complete.

\section{Main results}

First, we introduce some notations. Let $\Lambda_{\lambda}$ be the Nehari manifold associated with $\left(1.1_{\lambda}\right)-\left(1.2_{\lambda}\right)$; i.e.

$$
\Lambda_{\lambda}:=\left\{(u, v) \in Z:\left\langle I_{\lambda}^{\prime}(u, v),(u, v)\right\rangle=0\right\} .
$$

It is clear that $\Lambda_{\lambda}$ is closed in $Z$ and all critical points of $I_{\lambda}$ must lie on $\Lambda_{\lambda}$. So, $(u, v) \in \Lambda_{\lambda}$ if and only if

$$
\begin{aligned}
\int_{\Omega} \nu_{1}(x)|\nabla u|^{p} d x-\lambda \int_{\Omega} a(x)|u|^{p} d x & -\lambda \int_{\Omega} b(x)|u|^{\alpha+1}|v|^{\beta+1} d x \\
& =\frac{1}{(\alpha+1)(\delta+1)} \int_{\Omega} \mu(x)|u|^{\gamma+1}|v|^{\delta+1} d x \\
\int_{\Omega} \nu_{2}(x)|\nabla v|^{q} d x-\lambda \int_{\Omega} d(x)|v|^{q} d x & -\lambda \int_{\Omega} b(x)|u|^{\alpha+1}|v|^{\beta+1} d x \\
& =\frac{1}{(\beta+1)(\gamma+1)} \int_{\Omega} \mu(x)|u|^{\gamma+1}|v|^{\delta+1} d x .
\end{aligned}
$$


Now, we define the following disjoint subsets of $\Lambda_{\lambda}$ :

$$
\begin{aligned}
\Lambda_{\lambda}^{+}:=\left\{(u, v) \in \Lambda_{\lambda}: \int_{\Omega} \mu(x)|u|^{\lambda+1}|v|^{\delta+1} d x<0\right\}, \\
\Lambda_{\lambda}^{0}:=\left\{(u, v) \in \Lambda_{\lambda}: \int_{\Omega} \mu(x)|u|^{\lambda+1}|v|^{\delta+1} d x=0\right\}, \\
\Lambda_{\lambda}^{-}:=\left\{(u, v) \in \Lambda_{\lambda}: \int_{\Omega} \mu(x)|u|^{\lambda+1}|v|^{\delta+1} d x>0\right\} .
\end{aligned}
$$

Note that the condition $\left(\Upsilon_{5}\right)$ implies that $\left(u_{1}, v_{1}\right) \notin \Lambda_{\lambda}^{-}$.

LEMma 3.1. The solution branch $C$ bends to the right of $\lambda_{1}$ at $\left(\lambda_{1}, 0,0\right)$; i.e. there exists $\rho>0$, such that $(\lambda, u, v) \in C$ and $\|u\|_{1, p}+\|v\|_{1, q}<\rho$, implies $\lambda>\lambda_{1}$.

Proof. Suppose not. Then, there exists a sequence $\left(\lambda_{n}, u_{n}, v_{n}\right) \in C$, such that $\left(u_{n}, v_{n}\right) \rightarrow 0$ in $Z, \lambda_{n} \leq \lambda_{1}, \lambda_{n} \rightarrow \lambda_{1}$ and

$$
\begin{aligned}
\int_{\Omega} \nu_{1}(x)\left|\nabla u_{n}\right|^{p} d x & -\lambda_{n} \int_{\Omega} a(x)\left|u_{n}\right|^{p} d x-\lambda_{n} \int_{\Omega} b(x)\left|u_{n}\right|^{\alpha+1}\left|v_{n}\right|^{\beta+1} d x \\
& =\frac{1}{(\alpha+1)(\delta+1)} \int_{\Omega} \mu(x)\left|u_{n}\right|^{\gamma+1}\left|v_{n}\right|^{\delta+1} d x, \\
\int_{\Omega} \nu_{2}(x)\left|\nabla v_{n}\right|^{q} d x & -\lambda_{n} \int_{\Omega} d(x)\left|v_{n}\right|^{q} d x-\lambda_{n} \int_{\Omega} b(x)\left|u_{n}\right|^{\alpha+1}\left|v_{n}\right|^{\beta+1} d x \\
& =\frac{1}{(\beta+1)(\gamma+1)} \int_{\Omega} \mu(x)\left|u_{n}\right|^{\gamma+1}\left|v_{n}\right|^{\delta+1} d x .
\end{aligned}
$$

We introduce the sequences $\widetilde{u}_{n}$ and $\widetilde{v}_{n}$ in the following way:

$$
\widetilde{u}_{n}=\frac{u_{n}}{\left(\left\|u_{n}\right\|_{1, p}^{p}+\left\|v_{n}\right\|_{1, q}^{q}\right)^{1 / p}} \quad \text { and } \quad \widetilde{v}_{n}=\frac{v_{n}}{\left(\left\|u_{n}\right\|_{1, p}^{p}+\left\|v_{n}\right\|_{1, q}^{q}\right)^{1 / q}}
$$

which are bounded sequences. Indeed, we have

$$
\left\|\widetilde{u}_{n}\right\|_{1, p}^{p}+\left\|\widetilde{v}_{n}\right\|_{1, q}^{q}=1 \quad \text { for every } n \in \mathbb{N} \text {. }
$$

Thus, we may assume $\left(\widetilde{u}_{n}, \widetilde{v}_{n}\right) \rightarrow\left(\widetilde{u}_{0}, \widetilde{v}_{0}\right)$ in $Z$. Using $(\alpha+1) / p+(\beta+1) / q=1$ in the condition $(\mathcal{H})$, we have

$$
\int_{\Omega} b(x)\left|\widetilde{u}_{n}\right|^{\alpha+1}\left|\widetilde{v}_{n}\right|^{\beta+1} d x=\frac{\int_{\Omega} b(x)\left|u_{n}\right|^{\alpha+1}\left|v_{n}\right|^{\beta+1} d x}{\left\|u_{n}\right\|_{1, p}^{p}+\left\|v_{n}\right\|_{1, q}^{q}} .
$$

Moreover, the range of exponents and Lemma 2.1 implies

$$
\frac{\int_{\Omega} \mu(x)\left|u_{n}\right|^{\gamma+1}\left|v_{n}\right|^{\delta+1} d x}{\left\|u_{n}\right\|_{1, p}^{p}+\left\|v_{n}\right\|_{1, q}^{q}} \leq \frac{\|\mu\|_{\omega_{2}}\left\|u_{n}\right\|_{p^{*}}^{\gamma+1}\left\|v_{n}\right\|_{q^{*}}^{\delta+1}}{\left\|u_{n}\right\|_{1, p}^{p}+\left\|v_{n}\right\|_{1, q}^{q}} \rightarrow 0,
$$


as $\left(u_{n}, v_{n}\right) \rightarrow 0$ in $Z$. Using (3.1) and (3.2), we obtain

$$
\begin{aligned}
& \int_{\Omega}\left(\nu_{1}(x)\left|\nabla \widetilde{u}_{n}\right|^{p}-\lambda_{n} a(x)\left|\widetilde{u}_{n}\right|^{p}-\lambda_{n} b(x)\left|\widetilde{u}_{n}\right|^{\alpha+1}\left|\widetilde{v}_{n}\right|^{\beta+1}\right) d x \rightarrow 0, \\
& \int_{\Omega}\left(\nu_{2}(x)\left|\nabla \widetilde{v}_{n}\right|^{q}-\lambda_{n} d(x)\left|\widetilde{v}_{n}\right|^{q}-\lambda_{n} b(x)\left|\widetilde{u}_{n}\right|^{\alpha+1}\left|\widetilde{v}_{n}\right|^{\beta+1}\right) d x \rightarrow 0,
\end{aligned}
$$

as $n \rightarrow \infty$. Moreover, the compactness of the operators $D$ and $B$ implies that

$$
\begin{aligned}
\lambda_{n} \int_{\Omega} a(x)\left|\widetilde{u}_{n}\right|^{p} d x & \rightarrow \lambda_{1} \int_{\Omega} a(x)\left|\widetilde{u}_{0}\right|^{p} d x, \\
\lambda_{n} \int_{\Omega} d(x)\left|\widetilde{v}_{n}\right|^{q} d x & \rightarrow \lambda_{1} \int_{\Omega} d(x)\left|\widetilde{v}_{0}\right|^{q} d x, \\
\lambda_{n} \int_{\Omega} b(x)\left|\widetilde{u}_{n}\right|^{\alpha+1}\left|\widetilde{v}_{n}\right|^{\beta+1} d x & \rightarrow \lambda_{1} \int_{\Omega} b(x)\left|\widetilde{u}_{0}\right|^{\alpha+1}\left|\widetilde{v}_{0}\right|^{\beta+1} d x,
\end{aligned}
$$

as $n \rightarrow \infty$. Hence, $\left(\widetilde{u}_{n}, \widetilde{v}_{n}\right) \rightarrow\left(\widetilde{u}_{0}, \widetilde{v}_{0}\right) \neq(0,0)$, since $\left\|\left(\widetilde{u}_{n}, \widetilde{v}_{n}\right)\right\|_{Z}=1$, for every $n \in \mathbb{N}$. Also, $\left(\widetilde{u}_{0}, \widetilde{v}_{0}\right)$ is a solution of $\left(1.4_{\lambda_{1}}\right)-\left(1.5_{\lambda_{1}}\right)$. By Theorem $2.4(\mathrm{a})$, $\lambda_{1}$ is simple. Thus, $\widetilde{u}_{0}=k^{p} u_{1}$ and $\widetilde{v}_{0}=k^{q} v_{1}$, for some positive constant $k$. Multiplying equations (3.1) and (3.2) by $(\alpha+1) / p$ and $(\beta+1) / q$, respectively, adding the resulting equations, and using condition $(\mathcal{H})$, we deduce that

$$
A_{\lambda_{n}}\left(u_{n}, v_{n}\right)=c_{1} \int_{\Omega} \mu(x)\left|u_{n}\right|^{\gamma+1}\left|v_{n}\right|^{\delta+1} d x, \quad \text { for any } n \in \mathbb{N},
$$

where $c_{1}=1 /(p(\delta+1))+1 /(q(\gamma+1))$. From the variational characterization (2.1) of the eigenvalue $\lambda_{1}$, equation (3.4), and condition $\left(\Upsilon_{5}\right)$ we conclude that

$$
0 \leq \lim _{n \rightarrow \infty} c_{1} \int_{\Omega} \mu(x)\left|\widetilde{u}_{n}\right|^{\gamma+1}\left|\widetilde{v}_{n}\right|^{\delta+1} d x=c_{2} \int_{\Omega} \mu(x)\left|u_{1}\right|^{\gamma+1}\left|v_{1}\right|^{\delta+1} d x<0,
$$

for some $c_{2}=c_{2}\left(c_{1}, k\right)>0$, which is a contradiction, and so the proof is complete.

Corollary 3.2. Suppose that $(\lambda, u, v) \in C$, such that $(\lambda, u, v)$ is close enough to $\left(\lambda_{1}, 0,0\right)$; then $(u, v) \in \Lambda_{\lambda}^{+}$.

Proof. Let $\left(\lambda_{n}, u_{n}, v_{n}\right) \in C$, such that $\left(u_{n}, v_{n}\right) \rightarrow(0,0)$ in $Z$ and $\lambda_{n} \rightarrow \lambda_{1}$. Then, using the same arguments as in Lemma 3.1 we may prove that

$$
\int_{\Omega} \mu(x)\left|u_{n}\right|^{\gamma+1}\left|v_{n}\right|^{\delta+1} d x<0, \quad \text { for } n \text { large enough; }
$$

i.e. $\left(u_{n}, v_{n}\right) \in \Lambda_{\lambda}^{+}$, when $n$ is large enough.

Lemma 3.3. There exists $\lambda^{0}>\lambda_{1}$, such that for every $\lambda \in\left(\lambda_{1}, \lambda^{0}\right)$ the set $\Lambda_{\lambda}^{-}$is closed in $Z$. 
Proof. First note that $\Lambda_{\lambda}^{-} \neq \emptyset$, since $\mu^{+} \not \equiv 0$. We have to prove that for any $\left(u_{n}, v_{n}\right) \in \Lambda_{\lambda}^{-}$such that $\left(u_{n}, v_{n}\right) \rightarrow(u, v)$ in $Z$, we have $(u, v) \in \Lambda_{\lambda}^{-}$, when $\lambda \in\left(\lambda_{1}, \lambda^{0}\right)$. Due to the compactness of the operator $M$, this will be the case if

$$
\int_{\Omega} \mu(x)\left|\widetilde{u}_{n}\right|^{\gamma+1}\left|\widetilde{v}_{n}\right|^{\delta+1} d x \rightarrow \int_{\Omega} \mu(x)|u|^{\gamma+1}|v|^{\delta+1} d x>0 .
$$

Assume that such a $\lambda^{0}$ does not exist. Then, there exists a sequence $\left(\lambda_{n}, u_{n}, v_{n}\right)$, with $\left(u_{n}, v_{n}\right) \in \Lambda_{\lambda}^{-}$, such that

$$
\lambda_{n} \rightarrow \lambda_{1} \quad \text { and } \quad \int_{\Omega} \mu(x)\left|u_{n}\right|^{\gamma+1}\left|v_{n}\right|^{\delta+1} d x \rightarrow 0
$$

Since $\left(u_{n}, v_{n}\right)$ is a solution for the system $\left(1.1_{\lambda_{n}}\right)-\left(1.2_{\lambda_{n}}\right)$, we have that

$$
\begin{gathered}
\int_{\Omega}\left(\nu_{1}(x)\left|\nabla u_{n}\right|^{p}-\lambda_{n} a(x)\left|u_{n}\right|^{p}-\lambda_{n} b(x)\left|u_{n}\right|^{\alpha+1}\left|v_{n}\right|^{\beta+1}\right) d x \rightarrow 0, \\
\int_{\Omega}\left(\nu_{2}(x)\left|\nabla \widetilde{v}_{n}\right|^{q}-\lambda_{n} d(x)\left|v_{n}\right|^{q}-\lambda_{n} b(x)\left|u_{n}\right|^{\alpha+1}\left|v_{n}\right|^{\beta+1}\right) d x \rightarrow 0,
\end{gathered}
$$

as $n \rightarrow \infty$. Similar to Lemma 3.1, we may prove that the sequences $\left\{\widetilde{u}_{n}\right\}$ and $\left\{\widetilde{v}_{n}\right\}$ converge strongly to some $\left(\widetilde{u}_{0}, \widetilde{v}_{0}\right)$, and we have $\widetilde{u}_{0}=k^{p} u_{1}$ and $\widetilde{v}_{0}=k^{q} v_{1}$, for some positive constant $k$. The compactness of the operator $M$ implies that

$$
0 \leq \lim _{n \rightarrow \infty} c_{3} \int_{\Omega} \mu(x)\left|\widetilde{u}_{n}\right|^{\gamma+1}\left|\widetilde{v}_{n}\right|^{\delta+1} d x=c_{4} \int_{\Omega} \mu(x)\left|u_{1}\right|^{\gamma+1}\left|v_{1}\right|^{\delta+1} d x<0,
$$

for some positive constants $c_{3}$ and $c_{4}$, which is a contradiction. Thus, $\Lambda_{\lambda}^{-}$is closed in $Z$.

LEMma 3.4. The functional $I_{\lambda}$ satisfies the (PS) condition on $\Lambda_{\lambda}^{-}$, whenever $\lambda$ is close enough to $\lambda_{1}$.

Proof. Let the sequence $\left(u_{n}, v_{n}\right) \in \Lambda_{\lambda}^{-}$be such that $I_{\lambda}\left(u_{n}, v_{n}\right) \leq c$ and $I_{\lambda}^{\prime}\left(u_{n}, v_{n}\right) \rightarrow 0$, as $n \rightarrow \infty$. We first prove that $\left(u_{n}, v_{n}\right)$ is a bounded sequence. The quantity $M\left(u_{n}, v_{n}\right)$ is bounded, for all $n \in \mathbb{N}$, since

$$
\begin{aligned}
I_{\lambda}\left(u_{n}, v_{n}\right)-\left\langle I_{\lambda}^{\prime}\left(u_{n}, v_{n}\right),\left(\frac{u_{n}}{p}, \frac{v_{n}}{q}\right)\right\rangle \\
=\left[\frac{1}{p(\delta+1)}+\frac{1}{q(\gamma+1)}-\frac{1}{(\gamma+1)(\delta+1)}\right] M\left(u_{n}, v_{n}\right) .
\end{aligned}
$$

Therefore, $A_{\lambda}\left(u_{n}, v_{n}\right)$ must be bounded, too. Next, we claim that there exists a positive constant $\sigma$, such that

$$
\frac{A_{\lambda}\left(u_{n}, v_{n}\right)}{\left\|u_{n}\right\|_{1, p}^{p}+\left\|v_{n}\right\|_{1, q}^{q}} \geq \sigma>0, \quad \text { for every } n \in \mathbb{N},
$$


which would imply the boundedness of $\left(u_{n}, v_{n}\right)$ in $Z$. Suppose not. Then, there exists a sequence $\left(\lambda_{n}, u_{n}, v_{n}\right)$, with $\left(u_{n}, v_{n}\right) \in \Lambda_{\lambda}^{-}$, such that $\lambda_{n} \rightarrow \lambda_{1}$ and

$$
\frac{A_{\lambda_{n}}\left(u_{n}, v_{n}\right)}{\left\|u_{n}\right\|_{1, p}^{p}+\left\|v_{n}\right\|_{1, q}^{q}}=A_{\lambda_{n}}\left(\widetilde{u}_{n}, \widetilde{v}_{n}\right) \rightarrow 0
$$

where $\left(\widetilde{u}_{n}, \widetilde{v}_{n}\right)$ are the sequences introduced by (3.3). The boundedness of $\left(\widetilde{u}_{n}, \widetilde{v}_{n}\right)$ implies that

$$
\left(\widetilde{u}_{n}, \widetilde{v}_{n}\right) \rightarrow\left(\widetilde{u}_{0}, \widetilde{v}_{0}\right)
$$

in $Z$, for some $\left(\widetilde{u}_{0}, \widetilde{v}_{0}\right) \in Z$. From the variational characterization (2.1) of $\lambda_{1}$ and By the weak lower semicontinuity of $A_{\lambda}$ we have

$$
0 \leq A_{\lambda_{1}}\left(\widetilde{u}_{0}, \widetilde{v}_{0}\right) \leq \liminf _{n \rightarrow \infty} A_{\lambda_{n}}\left(\widetilde{u}_{n}, \widetilde{v}_{n}\right)=0 .
$$

We claim that $\left(\widetilde{u}_{0}, \widetilde{v}_{0}\right) \neq(0,0)$. Otherwise, from the compactness of the functionals $D$ and $B$ we have

$$
\lim _{n \rightarrow \infty} D\left(\widetilde{u}_{n}, \widetilde{v}_{n}\right)=\lim _{n \rightarrow \infty} B\left(\widetilde{u}_{n}, \widetilde{v}_{n}\right)=0 .
$$

Hence, from (3.5) we conclude that $\left(\widetilde{u}_{n}, \widetilde{v}_{n}\right) \rightarrow(0,0)$ in $Z$, which contradicts the fact that $\left\|\left(\widetilde{u}_{n}, \widetilde{v}_{n}\right)\right\|_{Z}=1$, for every $n \in \mathbb{N}$.

Now, from (3.5) we must have that $\widetilde{u}_{0}=k^{p} u_{1}$ and $\widetilde{v}_{0}=k^{q} v_{1}$, for some positive constant $k$. Then from $\left(\Upsilon_{5}\right)$ we have

$$
0<\int_{\Omega} \mu(x)\left|\widetilde{u}_{n}\right|^{\gamma+1}\left|\widetilde{v}_{n}\right|^{\delta+1} d x \rightarrow c_{5} \int_{\Omega} \mu(x)\left|u_{1}\right|^{\gamma+1}\left|v_{1}\right|^{\delta+1} d x<0,
$$

which is a contradiction. Thus $\left(u_{n}, v_{n}\right)$ is a bounded sequence. Using the compactness of the functionals $D, B$, and $M$ and following the procedure from [12, Lemma 2.3] we obtain that $\left(u_{n}, v_{n}\right)$ has a convergent subsequence, and so the proof is complete.

Our main result is the following theorem.

Theorem 3.5. There exists $\lambda^{*}>\lambda_{1}$, such that the system $\left(1.1_{\lambda}\right)-\left(1.2_{\lambda}\right)$ has two nonnegative nonsemitrivial solutions, for every $\lambda \in\left(\lambda_{1}, \lambda^{*}\right)$.

Proof. By Theorem 2.6 and Corollary 3.2, there exist a nonsemitrivial solution for the system $\left(1.1_{\lambda}\right)-\left(1.2_{\lambda}\right)$, which belongs in $\Lambda_{\lambda}^{+}$. We prove the existence of a solution, which belongs in $\Lambda_{\lambda}^{-}$. Consider the set $\Lambda_{\lambda}^{-}$equipped with the metric $d\left(\widetilde{z}_{1}, \widetilde{z}_{2}\right)=\left\|\widetilde{z}_{1}-\widetilde{z}_{2}\right\|_{Z}$, for every $\widetilde{z}_{1}$ and $\widetilde{z}_{2}$ in $\Lambda_{\lambda}^{-}$. It is clear from Lemma 3.3, that for $\lambda^{*}$ close to $\lambda_{1}$, the set $\Lambda_{\lambda}^{-}$becomes a complete metric space. Using condition $(\mathcal{H})$, we observe that

$$
\begin{aligned}
A_{\lambda}(u, v)= & \frac{\alpha+1}{p} \int_{\Omega}\left(\nu_{1}(x)|\nabla u|^{p}-\lambda a(x)|u|^{p}-\lambda b(x)|u|^{\alpha+1}|v|^{\beta+1}\right) d x \\
& +\frac{\beta+1}{q} \int_{\Omega}\left(\nu_{2}(x)|\nabla v|^{q}-\lambda d(x)|v|^{q}-\lambda b(x)|u|^{\alpha+1}|v|^{\beta+1}\right) d x .
\end{aligned}
$$


Hence, for every $(u, v) \in \Lambda_{\lambda}$, using $(\alpha+1) / p+(\beta+1) / q=1$, we have

$$
I(u, v)=\left[\frac{1}{p(\delta+1)}+\frac{1}{q(\gamma+1)}-\frac{1}{(\gamma+1)(\delta+1)}\right] \int_{\Omega} \mu(x)|u|^{\gamma+1}|v|^{\delta+1} d x .
$$

Since $p<\gamma+1$ or $q<\delta+1$, we conclude that $I_{\lambda}(u, v)>0$ whenever $(u, v) \in \Lambda_{\lambda}^{-}$ and $I_{\lambda}$ is bounded below in $\Lambda_{\lambda}^{-}$, i.e.

$$
\inf _{(u, v) \in \Lambda_{\lambda}^{-}} I_{\lambda}(u, v) \geq 0 .
$$

On the other hand, the functional $I_{\lambda}$ satisfies the (PS) condition in $\Lambda_{\lambda}^{-}$(by Lemma 3.4). Thus, Ekeland's variational principle implies the existence of a solution for the system $\left(1.1_{\lambda}\right)-\left(1.2_{\lambda}\right)$. This solution is nonnegative, since $I_{\lambda}(|u|,|v|)=I_{\lambda}(u, v)$, and in addition, Lemma 2.7 implies that it is also nonsemitrivial.

Acknowledgements. The authors would like to thank the unknown referee for his/her valuable comments and suggestions.

\section{REFERENCES}

[1] L. Boccardo And D.G. De Figueiredo, Some remarks on a system of quasilinear elliptic equations, NoDEA Nonlinear Differential Equations Appl. 9 (2002), 309-323.

[2] G. Bonanno, S. Heidarkhani and D. O'Regan, Multiple solutions for a class of dirichlet quasilinear elliptic systems driven by a $(P, Q)$-Laplacian operator, Dynam. Systems Appl. 20 (2011), 89-100.

[3] Y. BozhKova And E. Mitidieri, Existence of multiple solutions for quasilinear systems via Fibering method, J. Differential Equations 190 (2003), 239-267.

[4] R. Dautray And J.L. Lions, Mathematical Analysis and Numerical Methods for Science and Technology, Vol. I: Physical Origins and Classical Methods, Springer-Verlag, Berlin, 1985.

[5] A. DJEllit AND A. TAS, Existence of solutions for a class of elliptic systems in $\mathbb{R}^{N}$ involving the p-Laplacian, Electron. J. Differential Equations 56 (2003), 1-8.

[6] P. DrábeK And Y.X. Huang, Multiple positive solutions of quasilinear elliptic equations in $\mathbb{R}^{N}$, Nonlinear Anal. 37 (1999), 457-466.

[7] P. Drábek, A. Kufner and F. Nicolosi, Quasilinear Elliptic Equations with Degenerations and Singularities, Walter de Gruyter \& Co., Berlin, 1997.

[8] P. Drábek, N.M. Stavrakakis and N.B. Zographopoulos, Multiple nonsemitrivial solutions for quasilinear elliptic systems, Differential Integral Equations 16 (2003), 15191531.

[9] A. KRIStÁLy, Existence of two non-trivial solutions for a class of quasilinear elliptic variational systems on strip-like domains, Proc. Edinb. Math. Soc. (2) 48 (2005), 465477.

[10] Z.-Q. OU AND C.-L. TANG, Existence and multiplicity of nontrivial solutions for quasilinear elliptic systems, J. Math. Anal. Appl. 383 (2011), 423-438.

[11] M.N. Poulou, N.M. Stavrakakis and N.B. Zographopoulos, Global bifurcation results on degenerate quasilinear elliptic systems, Nonlinear Anal. 66 (2007), 214-227.

[12] N.M. Stavrakakis and N.B. Zographopoulos, Existence results for some quasilinear elliptic systems in $\mathbb{R}^{N}$, Electron. J. Differential Equations 39 (1999), 1-15. 
[13] N.B. Zographopoulos, On the principal eigenvalue of degenerate quasilinear elliptic systems, Math. Nachr. 281 (9) (2008), 1351-1365.

Manuscript received November 28, 2012

Ghasem A. Afrouzi

Department of Mathematics

Faculty of Mathematical Sciences

University of Mazandaran

Babolsar, IRAN

E-mail address: afrouzi@umz.ac.ir

ARmin Hadjian

Department of Mathematics

Faculty of Basic Sciences

Iniversity of Bojnord

P.O. Box 1339

Bojnord 94531, IRAN

E-mail address: hadjian83@gmail.com

Nicolas B. Zographopoulos

University of Military Education

Hellenic Army Academy

Department of Mathematics and Engineering Sciences

Vari - 16673, Athens, GREECE

E-mail address: nzograp@gmail.com, zographopoulosn@sse.gr 